\title{
BMJ Open Kongcun Town Asymptomatic Intracranial Artery Stenosis study in Shandong, China: cohort profile
}

\author{
Xiang Wang, ${ }^{1}$ Yuanyuan Zhao, ${ }^{1}$ Xiaokang Ji, ${ }^{2}$ Shaowei Sang, ${ }^{3}$ Sai Shao, ${ }^{4}$ \\ Peng Yan, ${ }^{1}$ Shan Li, ${ }^{1}$ Jifeng Li, ${ }^{1}$ Guangbin Wang, ${ }^{4}$ Ming Lu, ${ }^{3}$ Yifeng Du, ${ }^{1}$ \\ Fuzhong Xue, ${ }^{2}$ Chengxuan Qiu, ${ }^{1,5}$ Qinjian Sun (i) ${ }^{1}$
}

To cite: Wang $X$, Zhao $Y$, Ji $X$, et al. Kongcun Town Asymptomatic Intracranial Artery Stenosis study in Shandong, China: cohort profile. BMJ Open 2020;10:e036454. doi:10.1136/ bmjopen-2019-036454

- Prepublication history for this paper is available online. To view these files, please visit the journal online (http://dx.doi org/10.1136/bmjopen-2019036454).

Received 16 December 2019 Revised 12 May 2020

Accepted 29 May 2020

\section{Check for updates}

(C) Author(s) (or their employer(s)) 2020. Re-use permitted under CC BY-NC. No commercial re-use. See rights and permissions. Published by BMJ.

${ }^{1}$ Department of Neurology, Shandong Provincial Hospital affiliated to Shandong First Medical University, Jinan, Shandong, China ${ }^{2}$ Department of Biostatistics, School of Public Health, Shandong University, Jinan, Shandong, China

${ }^{3}$ Department of Clinical Epidemiology, Qilu Hospital, Shandong University, Jinan, Shandong, China

${ }^{4}$ Department of Radiology, Shandong Medical Imaging Research Institute, Jinan,

Shandong, China

${ }^{5}$ Department of Neurobiology, Care Sciences and Society, Karolinska Universitetssjukhuset, Stockholm, Sweden

Correspondence to Dr Qinjian Sun; sqj1210@163.com

\section{ABSTRACT}

Purpose The population-based Kongcun Town

Asymptomatic Intracranial Artery Stenosis (KT-alCAS) study aims to investigate the prevalence of alCAS and major cardiovascular risk factors (CRFs) or biomarkers related to the development and prognosis of alCAS.

Participants The KT-alCAS study included 2311 rural residents who were aged $\geq 40$ years and living in Kongcun Town, Shandong Province, China. Baseline examination was conducted from October 2017 to October 2018, during which information on demographics, socioeconomics, personal and family medical history, and lifestyle factors was collected through face-to-face interviews, physical examination and blood tests. alCAS was initially screened using transcranial Doppler examination and then diagnosed using magnetic resonance angiography. Atherosclerosis in carotid arteries was diagnosed via carotid ultrasonography. High-resolution MRI was further used to evaluate the vessel wall of alCAS. Neuropsychological assessments were performed in the participants diagnosed with alCAS and the age-matched and sex-matched controls.

Findings to date of the 2311 participants, 2027 $(87.7 \%)$ completed the diagnostic procedure and alCAS was detected in 154 persons, resulting in an overall prevalence of $7.6 \%$. The prevalence of alCAS increased with advancing age from $5.1 \%$ in participants aged $40-49$ years to $12.7 \%$ in those aged $\geq 70$ years $(p<0.001)$. alCAS was detected in 305 intracranial arteries, including 221 $(72.5 \%)$ in the anterior circulation and $84(27.5 \%)$ in the posterior circulation $(p<0.001)$. In addition, major CRFs were highly prevalent among middle-aged and elderly rural dwellers who were free of clinical stroke.

Future plans Follow-up examinations will be performed every 3 years following the baseline examination. This study will increase our knowledge about the natural history of alCAS and facilitate studies of alCAS-associated disorders among rural-dwelling Chinese adults, such as ischaemic stroke and vascular cognitive impairment. Trial registration number ChiCTR1800017197.

\section{INTRODUCTION}

Intracranial artery stenosis (ICAS) due to atherosclerosis is a leading cause of ischaemic stroke worldwide, especially in Asian populations. ${ }^{1}$ In addition, intracranial atherosclerosis

\section{Strengths and limitations of this study}

This population-based study integrated transcranial Doppler (TCD) and magnetic resonance angiography to detect asymptomatic intracranial artery stenosis (alCAS) among residents living in rural communities, which is feasible to investigate prevalence of alCAS in rural residents.

- High-resolution MRI scans were used to further identify characteristics of alCAS.

- The use of TCD as a screening tool for alCAS might have missed some cases and underestimated the prevalence of alCAS.

- The study sample was selected from only one rural area, and thus, cautiousness is needed when generalising the study findings to other rural populations in China.

has been associated with cognitive impairment. $^{2}$ Atherosclerotic lesions may develop and progress silently over years, subsequently impair cognitive function or even suddenly cause clinical stroke. Therefore, the detection of ICAS in the asymptomatic phase may facilitate early preventive and therapeutic interventions, and thus delay the progression to symptomatic stroke and cognitive impairment. ${ }^{34}$ Several population-based studies that investigated the prevalence of asymptomatic ICAS (aICAS, ie, ICAS without a history of stroke or transient ischaemic attack (TIA)) by using non-invasive tools, such as transcranial ultrasound or magnetic resonance angiography (MRA), suggested that the prevalence of aICAS ranged from around $9 \%$ in Caucasian populations ${ }^{56}$ and $12 \%$ in African Americans ${ }^{6}$ to as high as $24.5 \%$ in Asian populations. ${ }^{7-10}$ The substantial racial differences in the prevalence and distribution of aICAS could be due partly to different genetic and environmental factors. ${ }^{11}$ Therefore, the study involving Asian populations who are more vulnerable to ICAS or aICAS may shed light 
on the effects of genetic backgrounds, circumstances or social customs, and habits difference on the development of ICAS or aICAS. Moreover, data remain limited with regard to the prevalence of aICAS among rural residents in China, where cardiovascular risk factors $(\mathrm{CRFs})$ are highly prevalent and poorly controlled. ${ }^{12}$

Furthermore, the natural history and prognosis of aICAS remains poorly understood. The Warfarin vs Aspirin Symptomatic Intracranial Disease trial showed that the risk of stroke from aICAS was relatively low among patients with coexisting symptomatic ICAS and aICAS. ${ }^{13}$ In addition, asymptomatic atherosclerotic stenosis of the middle cerebral artery (MCA) is apparently not a malignant disease. ${ }^{14}{ }^{15}$ These studies suggest that aICAS may have a benign long-term prognosis. It is worth noting that these previous studies have either focused on selective patient groups (co-existence of asymptomatic and symptomatic stenosis) or targeted special vessels (eg, MCA), and the general population-based studies that aimed at investigating the prognosis of aICAS remain scarce. Indeed, a population-based prospective study of 2807 healthy volunteers (mean age, 62.0 years) in Japan found that aICAS was a risk factor for clinical stroke. ${ }^{16}$ However, the predictors for the conversion and progression from aICAS to the symptomatic stage remain largely unclear.

Symptomatic ICAS (ie, ICAS with a history of stroke or TIA) is an independent risk factor for cognitive decline. However, whether aICAS is associated with subsequent cognitive decline remains to be explored. Recently, a clinical-based study suggested that in participants with asymptomatic severe MCA stenosis, poor collateral circulation was associated with declines in multiple cognitive domains characterised by vascular cognitive impairment. ${ }^{17}$ The association between cognitive function and aICAS in other intracranial arteries remains to be clarified.

Therefore, we initiated the Kongcun Town Asymptomatic Intracranial Artery Stenosis (KT-aICAS) study in Shandong Province, China. This cohort profile aimed to describe the establishment of the study cohort, baseline assessments and some of the findings from this cohort.

\section{COHORT DESCRIPTION}

\section{Study design and purposes}

KT-aICAS is designed as a population-based prospective cohort study. The purposes of the KT-aICAS were the following: (1) to investigate occurrence (eg, prevalence and incidence) and distribution of aICAS among middleaged and older adults living in rural communities; (2) to explore CRFs and clinical biomarkers of aICAS that may predict the progression from aICAS to ischaemic stroke; (3) to assess the association between aICAS and cognitive impairment and (4) to build a comprehensive database that facilitates longer-term multidisciplinary research regarding the biomedical, clinical, neuroimaging and epidemiological aspects of aICAS. At baseline, a cross-sectional study was conducted to detect the prevalence and distribution of aICAS. A subsequent follow-up assessment will be performed to determine the incidence rates of aICAS; examine the association between aICAS and ischaemic stroke; and explore a variety of CRFs, clinical features, imaging markers or biomarkers for the conversion from aICAS to symptomatic ICAS, clinical stroke or TIA and the potential cognitive consequences.

\section{Study communities and population}

The KT-aICAS study targeted all registered residents who were aged $\geq 40$ years, free of a history of stroke or TIA and living in the Kongcun Town of Pingyin county in Shandong Province in October 2017. Self-report of a physician diagnosis and/or clinical examinations showing typical symptoms were considered as a history of stroke or TIA, which was determined during the interviews and clinical examination. Kongcun Town is a rural township that is located $\sim 76 \mathrm{~km}$ west of Jinan, the capital of Shandong Province, China. Kongcun Town includes 230000 residents in an area of $\sim 5000$ hectares. The majority of people in the town are farmers. Since 2009, the School of Public Health at Shandong University has been working closely with the town government and local health professionals to conduct epidemiological surveys on health conditions of local residents every 3 years. This helped improve the cooperation from local residents (eg, a high participation rate at baseline and possibly high adherence to future follow-up assessments) for implementing this project. Following the baseline assessment in 2017-2018, all participants will be invited to undertake a follow-up assessment every 3 years with the aim of detecting patients with aICAS and identifying incident clinical stroke and cognitive impairment. Figure 1 shows the basic design of the KT-aICAS study and the flow chart of the study population and assessments.

\section{Ethics approval and consent to participate}

The protocol of the KT-aICAS study was reviewed and approved by the ethical standards committees on human experimentation at Shandong Provincial Hospital affiliated to Shandong First Medical University. Written informed consent was obtained from all of the participants. The study was conducted in accordance with the ethical principles for medical research involving human subjects expressed in the Declaration of Helsinki. The face-to-face interviews were scheduled at the most convenient times for the participants. Respondents were free to refuse participation or withdraw from the study at any time. Any case of serious illness that had not been detected previously was urgently referred to the local hospital for further examinations. All participant-related information was deidentified from the database to preserve privacy.

\section{Baseline assessments}

Baseline assessments were performed in October 2017 to October 2018, which included a two-phase design as described below. 


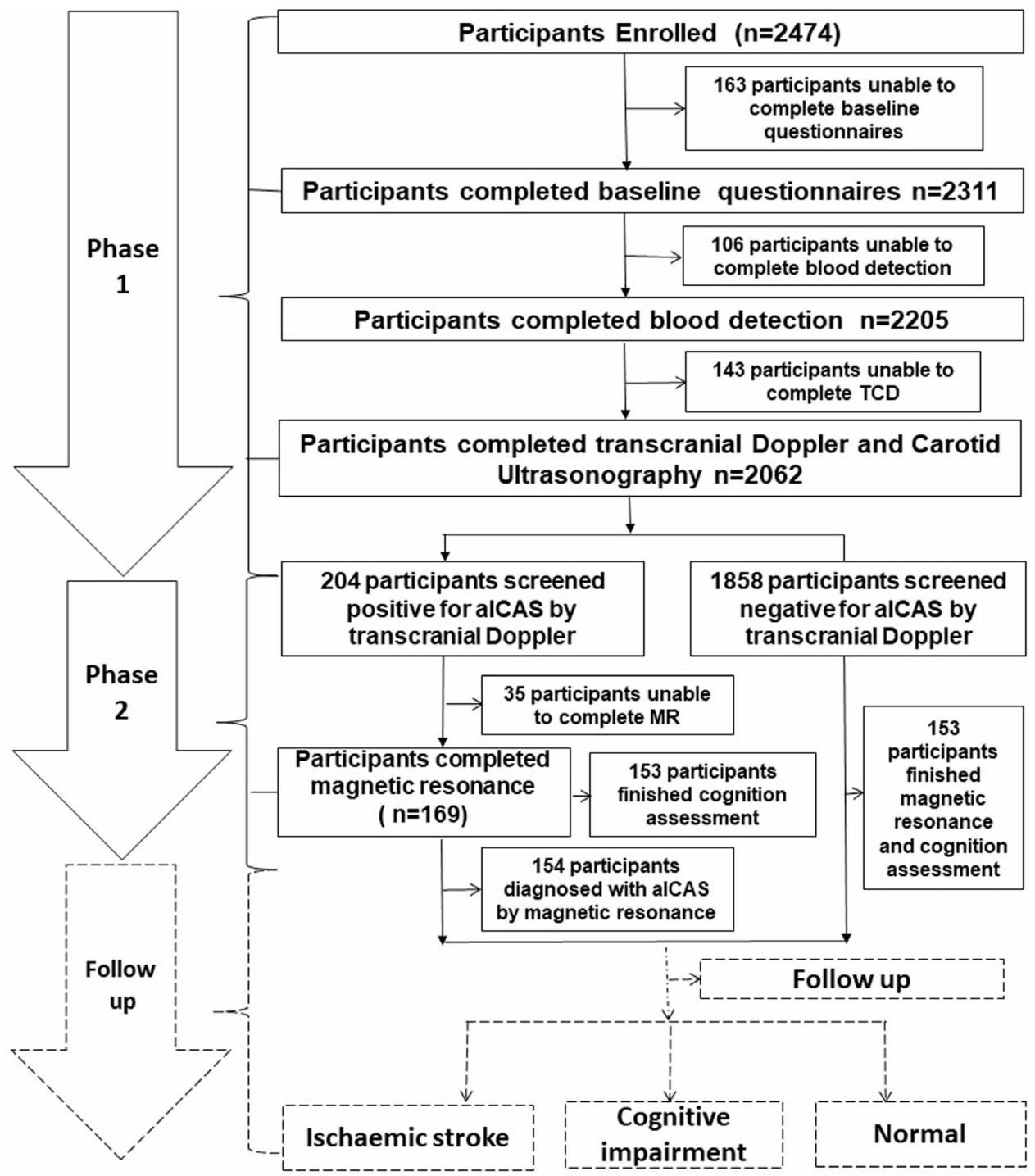

Figure 1 Flow chart of the study participants. alCAS, asymptomatic intracranial artery stenosis; MR, magnetic resonance; TCD, transcranial Doppler.

Phase 1

From October to November 2017, we performed a phase 1 assessment, a screening phase that aimed to collect baseline data and identify people at high risk of aICAS. During this phase, all participants underwent a structured questionnaire survey, a transcranial Doppler (TCD) examination and a colour Doppler ultrasound examination (neck) on the same day.

Face-to-face interviews. A standardised questionnaire was used to collect data on demographic features, health and family history (eg, chronic diseases), CRFs (eg, hypertension, diabetes and obesity), lifestyle factors (eg, diet habits, smoking, alcohol consumption, tea consumption and physical activity), menstrual and reproductive history, quality of life and current use of medication. ${ }^{18} 19$ Typically, the interview took approximately $30 \mathrm{~min}$. Then, all participants underwent physical and clinical examinations, which included blood pressure and anthropometric measurements, carotid artery B-mode ultrasonography and TCD examination.
Blood pressure and anthropometric measurements. All measurements were performed by trained nurses. After a 5 min rest, arterial blood pressure was measured twice at a 5 min interval using an automated sphygmomanometer with the participant in the seated position. The mean value of the two measurements was used in the analysis. Hypertension was defined as blood pressure $\geq 140 / 90 \mathrm{~mm}$ $\mathrm{Hg}$ or use of antihypertensive drugs. Height was measured without shoes using a standard right-angle device and a fixed measurement tape (to the nearest $0.5 \mathrm{~cm}$ ). Body weight without heavy clothing was measured. We defined obesity as a body mass index (BMI) $\geq 28 \mathrm{~kg} / \mathrm{m}^{2}$ and overweight as a BMI of $24-27.9 \mathrm{~kg} / \mathrm{m}^{2}$.

TCD ultrasound examination. Participants were examined with a portable machine (VIASYS Companion III, Nicolet, Washington, USA) by two experienced physicians with expertise in sonography after a 5 min rest in a quiet and comfortable room. The bilateral MCA, anterior cerebral artery (ACA), posterior cerebral artery (PCA), vertebral artery (VA) and basilar artery (BA) were examined 
with a $2 \mathrm{MHz}$ probe through a temporal window, occipital window and eye window, respectively. The depth, peak systolic velocity (PSV), mean flow velocity (MFV), end diastolic velocity (EDV) and pulsatility index were measured for each artery. The following MFV cut-offs were used for the diagnosis of $\geq 50 \%$ intracranial stenosis of various arteries according to the criteria described in the Stroke Outcomes and Neuroimaging of Intracranial Atherosclerosis (SONIA) $\operatorname{trial}^{20}: \mathrm{MFV} \geq 100 \mathrm{~cm} / \mathrm{s}$ for MCA stenosis, MFV $\geq 80 \mathrm{~cm} / \mathrm{s}$ for VA stenosis and MFV $\geq 80$ $\mathrm{cm} / \mathrm{s}$ for BA stenosis. Because the ACA and PCA were not evaluated in the SONIA trial, we followed previously validated criteria to define $\geq 50 \%$ stenosis in these vessels ${ }^{21}$ : $\mathrm{MFV} \geq 80 \mathrm{~cm} / \mathrm{s}$ and $\geq 30 \%$ different when compared with the contralateral ACA segment for ACA stenosis and MFV $\geq 80 \mathrm{~cm} / \mathrm{s}$ for PCA stenosis. The criteria for $\geq 70 \%$ stenosis were as follows ${ }^{22}$ : MCA:MFV $\geq 120 \mathrm{~cm} / \mathrm{s}$ or a stenotic/ prestenotic ratio $\geq 3$ or low velocity; VA and $\mathrm{BA}$ : MFV $\geq 110 \mathrm{~cm} / \mathrm{s}$ or a stenotic/prestenotic ratio $\geq 3$. In addition, the presence of turbulence or musical sounds and an abnormal segmental velocity were considered for the diagnosis of stenosis.

Carotid ultrasonography examination. Carotid ultrasound examination was performed in the head-straight flat supine position by two experienced physicians to measure intimal medial thickness (IMT), PSV and EDV of the common carotid artery (CCA), internal carotid artery (ICA) and external carotid artery with a 7-MHz linear transducer (Siemens ACUSON P500). First, the structure of the vascular wall of the full-length of the CCA and the bifurcation and the initial segment of the ICA as well as atherosclerotic plaques and intravascular echo were measured in a cross section. Then, the IMT of the distal segment of the CCA (1 to $1.5 \mathrm{~cm}$ below the bifurcation level), the internal diameter of the ICA and the distal segment of the CCA were measured in longitudinal sections. The diameter was defined as the vertical distance between the upper edge of the posterior wall of the vessel and the inferior border of the anterior wall. When vessel stenosis occurred, the residual diameter and original diameter were measured. The thickness of the IMT was defined as the vertical distance between the upper edge of the posterior wall of the vessel and the upper edge of the outer membrane. Location, morphology and integrity of the fibrous cap and the acoustic characteristics of the plaque were observed, and the size of the plaque was measured. Intimal thickening was defined as intimalmiddle film thickness $\geq 1.0 \mathrm{~mm}$, and plaque was defined as focal intimal-middle film thickness $\geq 1.5 \mathrm{~mm} .{ }^{23}$

Laboratory tests. After overnight fasting, participants came to the local clinic, and peripheral venous blood samples were taken in the morning. The blood was then allowed to clot in a $37^{\circ} \mathrm{C}$ water bath for 30 min before it was centrifuged at $3000 \mathrm{rpm}$ for $15 \mathrm{~min}$. Then, the serum supernatant was collected and separated into five aliquots $(200 \mu \mathrm{L}$ per aliquot) and immediately stored in liquid nitrogen. Blood tests were conducted at the certified clinical laboratory of the Shandong Provincial
Hospital Affiliated to Shandong University. We measured a complete blood count in the whole blood as well as serum fasting blood glucose (FBG), liver function, renal function, high-density lipoprotein-cholesterol (HDL-C), low-density lipoprotein-cholesterol (LDL-C), triglyceride, apolipoprotein genotyping, homocysteine, supersensitivity $\mathrm{C}$ reactive protein and insulin. The remaining serum samples were stored at $-80^{\circ} \mathrm{C}$ until further analyses. Diabetes was defined as $\mathrm{FBG} \geq 7.0 \mathrm{mmol} / \mathrm{L}$ or current use of blood glucose-lowering agents or insulin injection. High cholesterol was defined as total cholesterol $\geq 6.20 \mathrm{mmol} / \mathrm{L}$ or current use of hypolipidaemic drugs, and dyslipidaemia was defined as total cholesterol $\geq 6.20 \mathrm{mmol} / \mathrm{L}$, triglycerides $\geq 1.8 \mathrm{mmol} / \mathrm{L}, \mathrm{HDL}-\mathrm{C}<1.11$ $\mathrm{mmol} / \mathrm{L}$ or LDL-C $\geq 3.36 \mathrm{mmol} / \mathrm{L}$ or current use of hypolipidaemic drugs.

\section{Phase 2}

From April to October 2018, participants who were screened positive and a group of age-matched and sexmatched controls $(1: 1)$ who were screened negative for aICAS by TCD in phase 1 underwent phase 2 assessment in our hospital, which included neuropsychological assessments, structural brain MRI scans and MRA.

MRI protocol and acquisition. All invited participants underwent brain MRI scans on a 3.0T scanner (Achieve; Philips Medical Systems, Best, The Netherlands) with an eight-channel head coil. The core MRI protocol included MRA, dynamic susceptibility contrast-enhanced perfusionweighted imaging (PWI), diffusion tensor imaging (DTI), T1-weighted imaging with three-dimensional (3D) variable refocusing flip angle volume isotropic turbo spin-echo acquisition (3D T1VISTA), 3D T2VISTA, fluid-attenuated inversion recovery images and contrastenhanced T1VISTA. The post-enhanced T1VISTA parameters were the same as those used for pre-T1VISTA. A gadolinium contrast medium (Gd-DTPA, Magnevist; Beijing BeiLu Pharmaceutical, Beijing, China) was intravenously injected at the third acquisition $(0.2 \mathrm{~mL} / \mathrm{kg}$ or $4.5 \mathrm{~mL} / \mathrm{s}$ followed immediately by a $30 \mathrm{~mL}$ physiological saline flush). The extent of stenosis in the five evaluated arteries (bilateral MCA, bilateral intracranial ICA and BA) was classified into five grades by consensus as normal, mild (signal reduction $<50 \%$ ), moderate (signal reduction $\geq 50 \%$ and $<70 \%$ ), severe (signal reduction $\geq 70 \%$ ) or occlusion (focal signal loss with the presence of distal signal). The diagnosis and grading of stenosis was performed by a neurologist specialising in stroke and a clinical neuroradiologist.

Neuropsychological evaluation. Neuropsychological assessments were performed by experienced neuropsychologists trained specifically for this project and included tests of global cognitive function and specific cognitive domains. The Chinese versions of the MiniMental State Examination (MMSE) and the Montreal Cognitive Assessment (MoCA) were used to assess global cognitive function. ${ }^{24}$ Specific cognitive domains (eg, episodic memory, verbal fluency, attention and 
executive function) were assessed using the following neuropsychological tests: (1) the Rey Auditory Verbal Learning Test Learning (direct recall, delayed recall and word list recognition) for episodic memory, ${ }^{25}$ the Fuld Object-Memory Evaluation for object memory, ${ }^{26}$ (2) the Category Verbal Fluency test for verbal performance and executive function, ${ }^{27}$ (3) the Trail Making Test (A\&B) for executive function, ${ }^{28}(4)$ the Wechsler Memory Scale-III Forward Digit Span and Backward Digit Span for attention and executive function ${ }^{29}$ and (5) the Wechsler block design test and Clock Drawing Test for visuospatial and motor skills. ${ }^{30}$ In addition, depressive symptoms were assessed using the Hamilton Depression Scale. ${ }^{31}$ The Barthel Index was used to assess basic activities of daily living. ${ }^{32}$

Quality control procedure. Before the beginning of the study, all staff were centrally trained and required to demonstrate competency to perform different assessments, including interviews, biospecimen acquisition, cerebrovascular assessments and cognitive testing. Psychologists, neuroradiologists and neurologists attended a standardisation meeting to ensure the consistency of application of the evaluation criteria. The data collection and processing were checked and verified by at least two people, and the entry of the collected data was completed via integral double entry.

\section{Statistical analysis}

The data are presented as frequencies (\%) for categorical variables and as the mean (SD) or median (IQR) for continuous variables. Characteristics of the study participants by sex were compared using the general linear regression model for continuous variables and $\chi^{2}$ test for categorical variables. We describe the distribution of major CRFs and aICAS. We used the statistical packages R (http://www.r-project.org; V.3.4.3) and EmpowerStats (www.empowerstats.com; X\&Y Solutions) for all analyses.

\section{Follow-up assessments}

All survivors of the baseline participants will be invited to undertake a follow-up evaluation in the third year. The same questionnaire used for the baseline survey will be used during the follow-up examination for collecting epidemiological, neuropsychological and clinical data. Analysis of biomarkers as well as TCD test, carotid ultrasonography examination and brain MRI scan will also be performed.

\section{Patient and public involvement}

No patients or public were involved in the development of research questions and the design of the project. Participants were informed of the examination procedures and the main results of the examination, and contributed to the data used for this study. We plan to disseminate the key findings from the project among participants and local residents as part of health education and promotion programme.

\section{FINDINGS TO DATE}

In this ongoing prospective cohort study of middle-aged and older adults who were free of clinical stroke, we have demonstrated the feasibility of a two-phase procedure to study the epidemiology and nature history of aICAS in the remote rural communities by integrating epidemiological approaches with clinical, neuroimaging and neuropsychological techniques.

The characteristics of baseline participants in KT-aICAS by sex are reported in table 1. Compared with men, women were older, less educated, less likely to be single and had higher levels of total cholesterol, triglycerides, LDL-C and BMI, but lower levels of diastolic pressure and HDL-C. There was no significant sex difference in levels of FBG or systolic pressure and prevalence of hypertension. Men were much more likely to smoke and consume alcohol than women across all age groups, whereas women had a higher prevalence of diabetes, obesity and a higher cholesterol level than men mainly in groups over 60 years of age (figure 2). The sociocultural tradition in China, especially in rural regions, could explain the sex differences in behavioural factors such as smoking and alcohol consumption. ${ }^{33}$ In addition, the differences in health conditions between men and women might be partially attributable to sex differences in factors such as educational achievement, socioeconomic position and other unmeasured lifestyle factors (eg, diet). Taken together, our data showed that major CRFs were highly prevalent among the rural-dwelling middle-aged and elderly people in western Shandong Province, China, and that there were substantial sex differences in certain lifestyle factors. We have reported that these CRFs, especially when coexisting, are strongly correlated with aICAS. ${ }^{34}$ Among the subsample of people who received neuropsychological tests, men had a higher score than women in the MMSE test $(\mathrm{p}=0.001)$, but not in MoCA test $(\mathrm{p}=0.088)$, in which higher educational attainments in men than in women may partly contribute to the sex differences in the global cognitive tests because MMSE test is more sensitive to education than MoCA test. The potential cognitive consequence of CRFs and aICAS will be fully analysed in a future study. Therefore, given the well-established aetiological relationship of CRFs with atherosclerosis and clinical stroke in the literature as well as in findings from our study, active interventions to target these modifiable risk factors are urgently needed to counteract the huge burden of stroke in rural areas of China.

Of the 2311 participants, $2027(87.7 \%)$ completed the diagnostic procedure for aICAS. Out of the 2027 participants, $437(21.5 \%)$ participants have poor temporal window, and 154 persons (92 women and 62 men) were diagnosed with aICAS, which corresponded to the overall prevalence of $7.6 \%(8.7 \%$ in women vs $6.4 \%$ in men, $\mathrm{p}=0.055$ ). The overall prevalence of aICAS increased with advancing age, from $5.1 \%$ in individuals aged $40-49$ years to $12.7 \%$ in those aged $\geq 70$ years ( $p$ for trend $<0.001$ ) (figure 3). There was a sex difference in the patterns of age-specific prevalence of aICAS, such that the prevalence 
Table 1 Characteristics of the study participants by gender

\begin{tabular}{|c|c|c|c|c|}
\hline Characteristics $†$ & Total & Men & Women & P value* \\
\hline No of participants (\%) & $2311(100)$ & $1076(46.6)$ & $1235(53.4)$ & -- \\
\hline Age (years), mean (SD) & $57.6(10.5)$ & $56.3(10.3)$ & $58.8(10.5)$ & $<0.001$ \\
\hline Age group (years), n (\%) & & & & $<0.001$ \\
\hline $40-49$ & $613(26.6)$ & $353(32.9)$ & $260(21.1)$ & \\
\hline $50-59$ & $733(31.8)$ & $334(31.1)$ & $399(32.4)$ & \\
\hline $60-69$ & 607 (26.3) & $240(22.3)$ & $367(29.8)$ & \\
\hline$\geq 70$ & $352(15.3)$ & $147(13.7)$ & $205(16.7)$ & \\
\hline Marital statust, n (\%) & & & & $<0.001$ \\
\hline Married & $2058(89.1)$ & $1003(93.3)$ & $1055(85.4)$ & \\
\hline Single & $252(10.9)$ & $72(6.7)$ & $180(14.6)$ & \\
\hline Educational level†, n (\%) & & & & $<0.001$ \\
\hline Illiterate & $354(15.3)$ & $36(3.3)$ & $318(25.8)$ & \\
\hline Elementary school & $679(29.4)$ & $247(23.0)$ & $432(35.0)$ & \\
\hline Middle school & $976(42.3)$ & $582(54.1)$ & $394(31.9)$ & \\
\hline High school and above & $301(13.0)$ & $211(19.6)$ & $90(7.3)$ & \\
\hline Body mass index $\left(\mathrm{kg} / \mathrm{m}^{2}\right) \dagger$, mean (SD) & $25.2(3.8)$ & $24.8(3.2)$ & $25.5(4.1)$ & $<0.001$ \\
\hline Systolic pressure (mm Hg)†, mean (SD) & $145.0(22.4)$ & $144.5(21.3)$ & $145.4(23.4)$ & 0.365 \\
\hline Diastolic pressure $(\mathrm{mm} \mathrm{Hg}) \dagger$, mean (SD) & $88.3(12.7)$ & $89.9(13.0)$ & $86.9(12.2)$ & $<0.001$ \\
\hline Fasting blood glucose (mmol/L)†, mean (SD) & $6.1(1.8)$ & $6.0(1.8)$ & $6.1(1.9)$ & 0.084 \\
\hline LDL-C (mmol/L)†, mean (SD) & $3.0(0.7)$ & $2.9(0.7)$ & $3.1(0.7)$ & $<0.001$ \\
\hline HDL-C (mmol/L)†, mean (SD) & $1.6(0.4)$ & $1.6(0.4)$ & $1.6(0.3)$ & 0.031 \\
\hline Triglycerides (mmol/L) $†$, mean (SD) & $1.4(0.9)$ & $1.3(0.9)$ & $1.5(0.9)$ & $<0.001$ \\
\hline Total cholesterol (mmol/L)†, mean (SD) & $5.4(1.0)$ & $5.2(0.9)$ & $5.5(1.0)$ & $<0.001$ \\
\hline Dyslipidaemia†, n (\%) & $922(41.7)$ & $378(36.2)$ & $544(46.7)$ & $<0.001$ \\
\hline Smoking†, n (\%) & $504(21.9)$ & $489(45.6)$ & $15(1.2)$ & $<0.001$ \\
\hline Alcohol consumptiont, $\mathrm{n}(\%)$ & $727(31.5)$ & $672(62.5)$ & $55(4.5)$ & $<0.001$ \\
\hline Obesity†, n (\%) & 437 (19.2) & $165(15.5)$ & $272(22.5)$ & $<0.001$ \\
\hline Hypertension†, n (\%) & $1294(56.6)$ & $592(55.3)$ & $702(57.8)$ & 0.220 \\
\hline Diabetes†, n (\%) & 725 (32.9) & $319(30.5)$ & $406(34.9)$ & 0.028 \\
\hline Carotid stenosist, n (\%) & $59(2.9)$ & $33(3.4)$ & $26(2.5)$ & 0.202 \\
\hline alCAS†, n (\%) & $154(7.6)$ & $62(6.4)$ & 92 (8.6) & 0.055 \\
\hline MMSE score $\neq$, mean (SD) & $23.4(5.4)$ & $24.7(4.4)$ & $22.5(5.9)$ & 0.001 \\
\hline MoCA score $\neq$, mean (SD) & $17.9(5.3)$ & $18.6(4.8)$ & $17.5(5.6)$ & 0.088 \\
\hline
\end{tabular}

${ }^{*} \mathrm{P}$ values are for the test of differences between males and females.

†The number of participants with missing value were 1 for marital status and educational level, 38 for body mass index and obese status, 29 for blood pressure, 175 for fasting blood glucose, 106 for lipid measurements (LDL-C, HDL-C, triglycerides and total cholesterol), 8 for smoking, 2 for alcohol consumption, 102 for dyslipidaemia, 26 for hypertension, 104 for diabetes, 284 for carotid stenosis and 284 for alCAS (of the 2311 participants, 2027 completed the diagnostic procedure for alCAS).

‡MMSE and MoCA tests were performed in a subsample of 308 participants, including 154 with alCAS and 153 age-matched and sexmatched controls.

alCAS, asymptomatic intracranial artery stenosis; HDL-C, high-density lipoprotein cholesterol; LDL-C, low-density lipoprotein cholesterol; MMSE, Mini-Mental State Examination; MoCA, Montreal Cognitive Assessment.

increased steadily with age in men (from $2.8 \%$ in $40-49$ years of age to $12.9 \%$ in people aged $\geq 70$ years) ( $p$ for trend $<0.001)$, but in women the prevalence was relatively stable from 40 to 60 years of age (just over 8\%), and then substantially increased $(12.8 \%$ in people aged $\geq 70$ years) ( $p$ for trend=0.141). The reason for the sex differences is not fully understood, but the fast increase in the prevalence of aICAS with age after 60 years of age might be partially due to a decrease in oestrogen levels among postmenopausal women. ${ }^{35}$ Indeed, evidence has suggested that oestrogens have atheroprotective effect via anti-inflammation, protection of vascular endothelial 

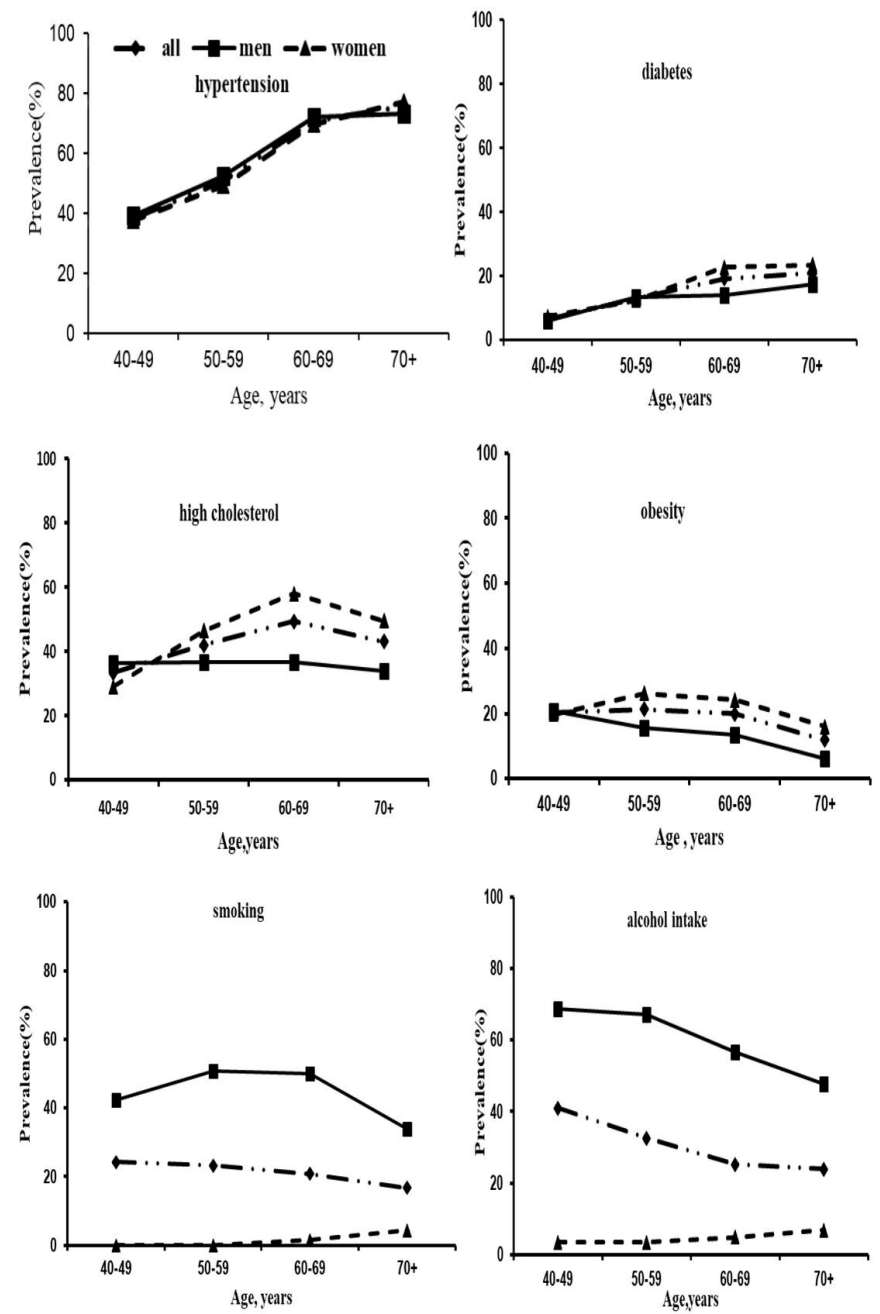

Figure 2 Age-specific and gender-specific prevalence (per 100 population) of cardiovascular risk factors.

cells and other mechanisms, ${ }^{3637}$ and that menopause has an unfavourable impact on several CRFs, structure of large arteries and atherosclerosis. ${ }^{38}$

A total of 305 intracranial arteries were diagnosed with aICAS; $221(72.5 \%)$ were involved in ACA, MCA or ICA, and $84(27.5 \%)$ were involved in PCA, VA or BA (table 2). The distributions of aICAS by regional arteries were as follows: ACA, $55(18.0 \%)$ arteries; MCA, $95(31.1 \%)$ arteries; ICA, $71(23.3 \%)$ arteries; PCA, 38

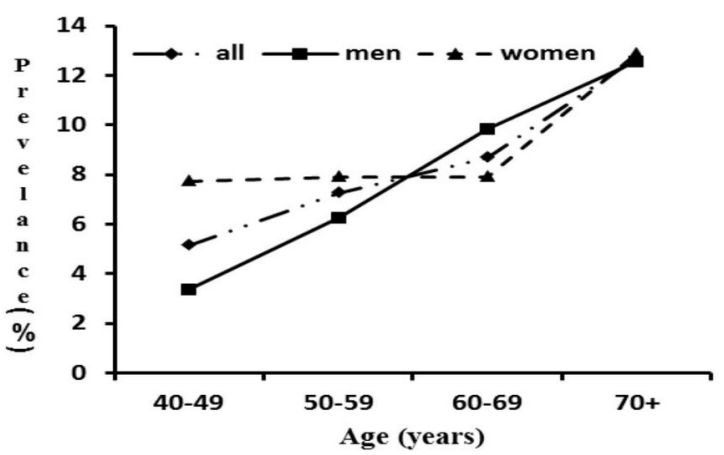

Figure 3 Age-specific and gender-specific prevalence (per 100 population) of asymptomatic intracranial artery stenosis.
(12.5\%) arteries; VA, $25(8.2 \%)$ arteries; BA, 21 (6.9\%) arteries. The arteries in the anterior circulation were more frequently involved than arteries in the posterior circulation.

Most previous studies have used either TCD or MRA alone to diagnose aICAS, and different detection approaches and procedures may contribute to the substantial variations in the reported prevalence of aICAS. For example, the prevalence of aICAS was reported to be $13.2 \%$ among coal mine workers aged $\geq 40$ years in a study that used only TCD to detect aICAS, whereas another study of people aged $\geq 40$ years (mean age, 58.1 years) from Korea reported that the prevalence of aICAS was $24.5 \%$ in all intracranial vessels. ${ }^{7}$ We used a two-phase approach that included a screening phase with TCD examination followed by a diagnostic phase with MRA, and we found that the overall prevalence of aICAS was $7.6 \%$ in this rural-dwelling middle-aged and elderly population. TCD examination, when used as a tool for detecting arterial stenosis, has the advantage of convenience and non-invasiveness but also has a high negative predictive value $(\sim 90 \%)$; thus, TCD is suitable for screening arterial stenosis in a large-scale population study. However, the positive predictive value of TCD examination is only $\sim 50 \%$. In contrast, MRA provides a good positive predictive value for ICAS but may not be suitable for large-scale community-based studies due to its high costs and inconvenience. ${ }^{20}$ In this study, we combined TCD with MRA to detect aICAS, which is feasible especially among people who are living in the remote rural communities. This approach will allow us to obtain a more accurate estimate of aICAS prevalence than that was achieved in previous studies which used either TCD or MRA alone.

We also found that the distribution of aICAS was more common in the anterior circulation than in the posterior circulation, in accordance with a study from Japan. ${ }^{39}$ The variations in the regional arterial distribution of aICAS are likely due to differences in the anatomical and haemodynamic characteristics between the anterior and posterior circulation. For example, in the anterior circulation, the higher number of vascular branches may increase the risk of atherosclerosis. ${ }^{40}$ In addition, TCD has relatively low sensitivity in detecting posterior circulation stenosis, ${ }^{41}$ and this may have contributed, in part, to the differences observed in the regional arterial distribution of aICAS.

In summary, in the community-based KT-aICAS study, aICAS affected nearly one-tenth of the rural-dwelling middle-aged and elderly residents, and its prevalence increased with advanced age. Anterior circulation arteries were most vulnerable to atherosclerotic lesions. In addition, major CRFs were highly prevalent among middleaged and elderly rural dwellers who were free of stroke. These findings may have important implications for the primary prevention of aICAS, clinical stroke and cognitive impairment. Future follow-up data from this cohort may contribute to the understanding of the epidemiology and natural history as well as cognitive consequences of aICAS among rural residents in China. 
Table 2 Arterial distribution of alCAS according to severity of stenosis (total number of arteries with alCAS=305)

\begin{tabular}{|c|c|c|c|c|c|}
\hline \multirow[b]{2}{*}{ Arteries } & \multicolumn{5}{|c|}{ Severity of intracranial arterial stenosis, $n(\%)$} \\
\hline & Mild & Moderate & Severe & Occlusion & Total \\
\hline ICA & $28(9.2)$ & $20(6.6)$ & $17(5.6)$ & $6(2.0)$ & $71(23.3)$ \\
\hline ACA & $22(7.2)$ & $17(5.6)$ & $16(5.3)$ & $0(0.0)$ & $55(18.0)$ \\
\hline PCA & $10(3.3)$ & $9(3.0)$ & $18(5.9)$ & $1(0.3)$ & $38(12.5)$ \\
\hline BA & $13(4.3)$ & $3(1.0)$ & $5(1.6)$ & $0(0.0)$ & $21(6.9)$ \\
\hline
\end{tabular}

ACA, anterior cerebral artery; alCAS, asymptomatic intracranial artery stenosis; BA, basilar artery; ICA, internal carotid artery; MCA, middle cerebral artery; PCA, posterior cerebral artery; VA, vertebral artery.

\section{STRENGTHS AND LIMITATIONS}

This cohort study targeted residents living in rural communities in China, to whom the research community has so far paid little attention. Second, we integrated TCD and MRA into a two-phase procedure to detect aICAS in a large-scale population study, and this approach is likely to allow us to obtain a relatively accurate estimate of the prevalence of aICAS. Furthermore, high-resolution MRI scans could help further evaluate the characteristics of arterial stenosis and avoid the contamination of ICAS cases with cases involving other non-atherosclerotic diseases. ${ }^{42}$ In addition, high-resolution MRI can be used to detect intraplaque haemorrhage, lipid cores and artery fibrous caps, thus providing further insight into the longterm prognosis of aICAS. To the best of our knowledge, this is the first community-based study of aICAS among an Asian population that used high-resolution MRI as well as PWI images and DTI images.

Our study also has limitations. First, we used TCD as a screening tool for arterial stenosis, which might have missed some cases of aICAS, and thus, have underestimated the overall prevalence of aICAS. Furthermore, in case of a poor or incomplete echo window for TCD, people without any stenosis in the VA, BA and ACA were considered to be non-stenotic. This might also have resulted in the underestimation of the true prevalence of aICAS in this population. Finally, our study sample was selected from only one rural area and the cohort might differ from other rural populations given that the local residents previously underwent health surveys, which should be kept in mind when generalising our findings to other rural populations.

Acknowledgements We thank all the study participants, the staff of the participating hospitals and the Steering Committee Members of the Kongcun Town study.

Contributors QS, FX and YD conceived the research question and designed the study. XW and FX conducted the statistical analysis. XW, CQ and QS wrote the first and subsequent drafts of the manuscript. XJ, SSa, SSh, YZ, PY, SL, JL, GW and $M L$ contributed to the epidemiological investigation. All authors contributed to data interpretation and critical revisions of the manuscript, and approved the final version of the submitted manuscript.

Funding This study was supported by the grants from Jinan Science and Technology Bureau (201704101), Department of Science and Technology of
Shandong Province (2014GSF118106, 2016GSF201062 and ZR2017MH114), the National Nature Science Foundation of China $(8171101298,81971128)$, the China Ministry of Sciences and Technology (2017YFC1310100 and 2017YFC0907003) and the International Science and Technology Cooperation Programme (2014DFA 32830). CQ received grants from the Swedish Research Council for the SinoSweden Network for Aging Research (2017-00740) and the Sino-Sweden Joint Research Project (2017-05819), Stockholm, Sweden, and the Taishan Overseas Scholar Program of Shandong Province, China.

Competing interests None declared.

Patient and public involvement Patients and/or the public were not involved in the design, conduct, reporting or dissemination plans of this research.

Patient consent for publication Not required.

Ethics approval Shandong Provincial Hospital affiliated to Shandong First Medical University in Jinan, Shandong, China (2017566).

Provenance and peer review Not commissioned; externally peer reviewed.

Data availability statement Data are available on reasonable request. Collaboration is welcomed and data sharing can be agreed on. The corresponding author can be contacted (sqj1210@163.com).

Open access This is an open access article distributed in accordance with the Creative Commons Attribution Non Commercial (CC BY-NC 4.0) license, which permits others to distribute, remix, adapt, build upon this work non-commercially, and license their derivative works on different terms, provided the original work is properly cited, appropriate credit is given, any changes made indicated, and the use is non-commercial. See: http://creativecommons.org/licenses/by-nc/4.0/.

ORCID iD

Qinjian Sun http://orcid.org/0000-0002-9323-9154

\section{REFERENCES}

1 Bos D, Portegies MLP, van der Lugt A, et al. Intracranial carotid artery atherosclerosis and the risk of stroke in whites: the Rotterdam study. JAMA Neurol 2014;71:405-11.

2 Beach TG, Wilson JR, Sue LI, et al. Circle of willis atherosclerosis: association with Alzheimer's disease, neuritic plaques and neurofibrillary tangles. Acta Neuropathol 2007;113:13-21.

3 Battistella V, Elkind M. Intracranial atherosclerotic disease. Eur J Neurol 2014;21:956-62.

4 Parfenov VA, Ostroumova OD, Ostroumova TM, et al. Vascular cognitive impairment: pathophysiological mechanisms, insights into structural basis, and perspectives in specific treatments. Neuropsychiatr Dis Treat 2019;15:1381-402.

5 López-Cancio E, Dorado L, Millán M, et al. The barcelonaasymptomatic intracranial atherosclerosis (Asia) study: prevalence and risk factors. Atherosclerosis 2012;221:221-5.

6 Suri MFK, Qiao Y, Ma X, et al. Prevalence of intracranial atherosclerotic stenosis using high-resolution magnetic resonance angiography in the general population: the atherosclerosis risk in communities study. Stroke 2016;47:1187-93.

7 Bae H-J, Lee J, Park J-M, et al. Risk factors of intracranial cerebral atherosclerosis among asymptomatics. Cerebrovasc Dis 2007;24:355-60. 
8 Wang A, Li Z, Luo Y, et al. Asymptomatic intracranial arterial stenosis and metabolic syndrome: the APAC study. PLoS One 2014;9:e113205.

9 Wong KS, Ng PW, Tang A, et al. Prevalence of asymptomatic intracranial atherosclerosis in high-risk patients. Neurology 2007;68:2035-8.

10 Huang HW, Guo MH, Lin RJ, et al. Prevalence and risk factors of middle cerebral artery stenosis in asymptomatic residents in Rongqi County, Guangdong. Cerebrovasc Dis 2007;24:111-5.

11 Arenillas JF, López-Cancio E, Wong KS. Biomarkers, natural course and prognosis. Front Neurol Neurosci 2016;40:93-108.

12 Song A, Liang Y, Yan Z, et al. Highly prevalent and poorly controlled cardiovascular risk factors among Chinese elderly people living in the rural community. Eur J Prev Cardiol 2014;21:1267-74.

13 Nahab F, Cotsonis G, Lynn M, et al. Prevalence and prognosis of coexistent asymptomatic intracranial stenosis. Stroke 2008;39:1039-41.

$14 \mathrm{Ni} \mathrm{J}$, Yao M, Gao S, et al. Stroke risk and prognostic factors of asymptomatic middle cerebral artery atherosclerotic stenosis. $J$ Neurol Sci 2011;301:63-5.

15 Lou X, Ma X, Liebeskind DS, et al. Collateral perfusion using arterial spin labeling in symptomatic versus asymptomatic middle cerebral artery stenosis. J Cereb Blood Flow Metab 2019;39:108-17.

16 Matsui R, Nakagawa T, Takayoshi H, et al. A prospective study of asymptomatic intracranial atherosclerotic stenosis in neurologically normal volunteers in a Japanese cohort. Front Neurol 2016;7:39.

17 Meng Y, Yu K, Zhang L, et al. Cognitive decline in asymptomatic middle cerebral artery stenosis patients with moderate and poor collaterals: a 2-year follow-up study. Med Sci Monit 2019;25:4051-8.

18 Kowal P, Chatterji S, Naidoo N, et al. Data resource profile: the world Health organization study on global ageing and adult health (SAGE). Int J Epidemiol 2012;41:1639-49.

19 Organization WH. The WHO STEPwise approach to noncommunicable disease risk factor surveillance (STEPS). 27. 20 Avenue Appia, 1211 Geneva 27, 2003.

20 Feldmann E, Wilterdink JL, Kosinski A, et al. The Stroke Outcomes and Neuroimaging of Intracranial Atherosclerosis (SONIA) trial. Neurology 2007;68:2099-106.

21 Tsivgoulis G, Sharma VK, Hoover SL, et al. Applications and advantages of power motion-mode doppler in acute posterior circulation cerebral ischemia. Stroke 2008;39:1197-204.

22 Zhao L, Barlinn K, Sharma VK, et al. Velocity criteria for intracranial stenosis revisited: an international multicenter study of transcranial doppler and digital subtraction angiography. Stroke 2011:42:3429-34.

23 Mozzini C, Roscia G, Casadei A, et al. Searching the perfect ultrasonic classification in assessing carotid artery stenosis: comparison and remarks upon the existing ultrasound criteria. $J$ Ultrasound 2016;19:83-90.

$24 \mathrm{Hu}$ J-bo, Zhou W-hua, Hu S-hua, et al. Cross-cultural difference and validation of the Chinese version of Montreal Cognitive Assessment in older adults residing in eastern China: preliminary findings. Arch Gerontol Geriatr 2013;56:38-43.
25 Brugnolo A, Morbelli S, Arnaldi D, et al. Metabolic correlates of Rey auditory verbal learning test in elderly subjects with memory complaints. J Alzheimers Dis 2014;39:103-13.

26 Anderson-Hanley C, Miele AS, Dunnam M. The fuld object-memory evaluation: development and validation of an alternate form. Appl Neuropsychol Adult 2013;20:1-6.

27 Whiteside DM, Kealey T, Semla M, et al. Verbal fluency: language or executive function measure? Appl Neuropsychol Adult 2016;23:29-34.

28 Misdraji EL, Gass CS. The TRAIL making test and its neurobehaviora components. J Clin Exp Neuropsychol 2010;32:159-63.

29 Giofrè D, Stoppa E, Ferioli P, et al. Forward and backward digit span difficulties in children with specific learning disorder. $J$ Clin Exp Neuropsychol 2016;38:478-86.

30 Sands LP, Terry H, Meredith W. Change and stability in adult intellectual functioning assessed by Wechsler item responses. Psychol Aging 1989;4:79-87.

31 Worboys $\mathrm{M}$. The hamilton rating scale for depression: The making of a "gold standard" and the unmaking of a chronic illness, 1960-1980. Chronic IIIn 2013;9:202-19.

32 Mlinac ME, Feng MC. Assessment of activities of daily living, selfcare, and independence. Arch Clin Neuropsychol 2016;31:506-16.

33 Wang S, Ungvari GS, Forester BP, et al. Gender differences in genera mental health, smoking, drinking and chronic diseases in older adults in Jilin Province, China. Psychiatry Res 2017;251:58-62.

34 Sun $\mathrm{Q}$, Wang $\mathrm{Q}$, Wang $X$, et al. Prevalence and cardiovascular risk factors of asymptomatic intracranial arterial stenosis: the Kongcun town study in Shandong, China. Eur J Neurol 2020;27:729-35.

35 Khalil RA, Estrogen Khalil RA:. Estrogen, vascular estrogen receptor and hormone therapy in postmenopausal vascular disease. Biochem Pharmacol 2013;86:1627-42.

36 Kassi E, Spilioti E, Nasiri-Ansari N, et al. Vascular inflammation and atherosclerosis: the role of estrogen receptors. Curr Med Chem 2015;22:2651-65.

37 Clarkson TB. Estrogen effects on arteries vary with stage of reproductive life and extent of subclinical atherosclerosis progression. Menopause 2018;25:1262-74.

38 Westendorp ICD, Grobbee DE, Witteman JCM. Oestrogen, atherosclerosis and cardiovascular disease in women: epidemiological studies on menopause and hormone replacement therapy. Neth Heart J 2001;9:177-81.

39 Uehara T, Tabuchi M, Mori E. Frequency and clinical correlates of occlusive lesions of cerebral arteries in Japanese patients without stroke. Evaluation by MR angiography. Cerebrovasc Dis 1998:8:267-72.

40 Arkuszewski M, Swiat M, Hurst RW, et al. Vertebral and basilar arteries: transcranial color-coded duplex ultrasonography versus conventional TCD in detection of narrowings. Neuroradiol $J$ 2012;25:509-14.

41 Xu W-H, Li M-L, Gao S, et al. Plaque distribution of stenotic middle cerebral artery and its clinical relevance. Stroke 2011;42:2957-9.

42 Jung SC, Kang DW, Turan TN. Vessel and vessel wall imaging. Front Neurol Neurosci 2016;40:109-23. 